\title{
Ketamine:An important drug with a serious adverse effect - a review
}

\begin{abstract}
Depression is a devastating mental disorder with a broad spectrum ranging from mild depression to major depressive disorder. There have been many drugs in use to treat depression that acts through different mechanisms. Most of the anti-depressant drugs will take weeks or months to take effect, and the discovery of the use of ketamine for depression has been widely studied. Ketamine is a glutamate -based antidepressant acts by blocking N-Methyl D-Aspartate receptors that show rapid antidepressant actions within hours of treatment, even useful to treat suicidal ideations. Ketamine enhances mTOR signaling, and this nonconventional mechanism offers novel approaches for the treatment of depression. Ketamine is also having shown to be effective to reduce post-operative pain and inflammation. In spite of all these benefits, ketamine has some serious adverse effects. Among them, ketamine cystitis is an important one especially in young population and requires attention towards its management. Dimethyl sulfoxide is a FDA approved drug used for bladder instillation. Further studies should be done to prevent and manage ketamine cystitis.
\end{abstract}

Keywords: Ketamine, bladder, ketamine cystitis, antidepressant, DMSO
Volume 9 Issue 6 - 2018

\author{
Roy Sebastian \\ Baylor Scott \& White Medical Center, USA
}

Correspondence: Roy Sebastian, BSW Medical CenterSunnyvale, 23I S.Collins Rd., Sunnyvale, TX 75|82, USA Tel 2149307527 Email roy.sabastian@usat.edu

Received: April 22, 2017 | Published: November 16, 2018
Abbreviations: CDC, Centers for Disease Control; NMDA, N-methyl-D-aspartate; EEG, Electroencephalogram; CNS, Central Nervous System; AMPA, alpha-amino-3-hydroxy-5-methyl-4isoxazole-propionic acid; KA, Kainate; GABA, Gamma-AminoButyric Acid; eEF2, eukaryotic elongation factor; BDNF, Brain, Derived Neurotrophic Factor; TNF, Tumor Necrosis Facyor; mTORC1, Mammalian Target of Rapamycin Complex 1; PFC, Prefrontal cortex; CRPS1, Complex regional pain syndrome 1; KC, Ketamine Cystitis; COX-2, Cyclooxygenase 2; iNOS, Inducible nitric oxide synthase; eNOS, endothelial Nitric Oxide Synthase; VUR, Vesico Ureteric Reflux (VUR); IC-Interstitial Cystitis; DMSO, Dimethylsulfoxide

\section{Introduction}

Major depressive disorder is one of the leading causes of disability in the United States with a statistically significant prevalence of 7.6\% (CDC, 2014). ${ }^{1}$ There are many treatment options available to treat depression, and antidepressant medications are the most commonly used. Most antidepressant drugs target the monoamine system and typically require four to six weeks to produce an antidepressant response. ${ }^{2}$ The efficacy of these medications is also limited, and a considerable proportion of the patients taking anti-depressants fail to achieve a sustained remission. ${ }^{3}$ There are few critical needs for antidepressants that are still unmet such as rapid onset of action; that increases the risk for suicide in patients who take antidepressants for major depressive disorder. All these drawbacks of traditional antidepressant medical therapy led to an urgent need for a rapidacting antidepressant with higher efficacy. Investigations for a better antidepressant medication pointed to an old anesthetic medication - ketamine, ${ }^{4}$ a noncompetitive glutamate N-methyl-D-aspartate (NMDA) receptor antagonist. Clinical data demonstrated that a small dose of ketamine could elicit a rapid antidepressant response in patients with major depression even the treatment-resistant depression and bipolar depression. ${ }^{5}$ Ketamine also has shown rapid anti-suicidal effects. ${ }^{6}$ Antidepressant effect of ketamine is not brought out by persistent blockade of NMDA receptors because of its short halflife of three hours; ${ }^{7}$ it could be the synaptic plasticity mechanisms or active metabolites of ketamine that are responsible for the longer term behavioral effects. Rapid and potent antidepressant effects were demonstrated after ketamine administration in patient groups known to respond poorly to current antidepressants, such as patients with the diagnosis of bipolar disorder and those with depressive symptoms that did not respond to electroconvulsive therapy. ${ }^{8}$ Ketamine has other important clinical implications too. Subanesthetic doses of ketamine potentiate opioid analgesia via NMDA receptor blockade. ${ }^{9}$ Studies show that perioperative ketamine improves postoperative pain and reduces need for opioids after surgery in a wide range of surgical procedures..$^{10}$ Ketamine has some serious adverse effects just like any other psychoactive drugs. It has an enormous potential for abuse. ${ }^{11}$ Chronic ketamine use has been associated with severe ketamine cystitis. ${ }^{12}$ This article reviews the benefits, risks, and management of adverse effects associated with ketamine.

\section{Ketamine: pharmacokinetics}

Ketamine is a hydrosoluble aryl-cyclo-alkylamine with a molecular mass of $238 \mathrm{~g} / \mathrm{mol}$ and a pKa 7.5 . Only $10-30 \%$ of the Ketamine binds to plasma protiens ${ }^{13}$ and has an extensive volume of distribution. Ketamine is mostly metabolized in norketamine $(80 \%)$ and 6 - hydroxy-norketamine $(15 \%)$ by a microsomal enzyme system ( $\mathrm{N}$-demethylation), finally excreted in bile and urine after conjugation in the liver by glucuronidation. This metabolism involves liver, kidneys, intestine and lungs. ${ }^{13}$ It has a half-life of three hours. Cytochrome P450 system is responsible for ketamine metabolism, and several microsomal enzymes are responsible for norketamine demethylation. ${ }^{14}$

\section{Norketamine pharmacokinetics}

Norketamine appears in blood 2-3 min after intravenous administration of ketamine bolus and reaches a peak about 30 min 
later. Norketamine has analgesic properties, and its power is about 20 $30 \%$ compared with ketamine. ${ }^{15}$ Though pharmacokinetics is not well known, the time plasma concentration analysis after a single ketamine administration demonstrates a slow elimination: norketamine remains in plasma for more than five hours after administration. ${ }^{13}$ Ketamine's elimination half-time is less than that of norketamine,${ }^{14}$ which strongly participates in the analgesic effect. Since norketamine accumulates, the need for ketamine decreases over time, when administered as continuous intravenous perfusion. ${ }^{13}$

\section{Administration routes}

Ketamine can be administered by intravenous infusion, intrathecal, epidural, intramuscular, intrarectal, intranasal or oral routes. Intravenous route has the advantage of faster binding with receptors in less than one minute where intramuscular route ensures high bioavailability of $93 \%$. Oral route has the least bioavailability of $20 \%$ because of the hepatic metabolism. ${ }^{13}$ After an intrathecal or epidural administration of ketamine, the drug goes into systemic circulation very rapidly and elicits a high-frequency psychodysleptic effects. ${ }^{13}$

\section{Pharmacodynamics}

Ketamine provides an entirely different state of anesthesia known as dissociative anesthesia compared with the effect produced by other anesthetic drugs 16 . In this state, the eyes stay open, with a typical nystagmus and conservation of laryngeal, corneal, and papillary reflexes with some muscle hypertonia. The anesthetic effect is compatible with the performance of a surgical procedure, with the absence of motor reaction orientated toward the nociceptive stimulus. ${ }^{16,17}$ Ketamine plasma concentrations of $200 \mathrm{ng} / \mathrm{ml}$ reduce pain scores by decreasing insular cortex and thalamus activities activated by a nociceptive stimulation ${ }^{17}$ and reduces pain perception by lowering the pain-induced cerebral activation in a dose-dependent manner. Ketamine alters electroencephalogram (EEG) differently from other anesthetic drugs by diminuting alpha rhythm amplitude with its abolition occasionally without modification of its frequency 18and the appearance of theta waves, without a measurable relationship with the depth of narcosis. Ketamine does not change the amplitude of mid-latency auditory evoked potentials or somatosensory evoked potentials. ${ }^{18}$

\section{Mechanisms of action}

\section{Neuropharmacology of ketamine is complex}

The primary mechanism of action is ketamine's action on glutamate binding sites, NMDA (N-Methyl-D-Aspartate) receptors, and non-NMDA receptors. ${ }^{19}$ Glutamate is the most abundant amino acid in the central nervous system (CNS), involving glutaminergic synapses. Liberation of glutamate activates several pre- and postsynaptic receptors located on ion channels. Ionotropic glutamate receptors are mainly categorized as NMDA (specifically activated by N-methyl-D-aspartate) and non-NMDA (such as AMPA [alphaamino-3-hydroxy-5-methyl-4-isoxazole-propionic acid] and KA [kainate]) receptors. NMDA receptors are present on almost all the cells in the CNS, particularly in the structures related to nociception, such as primary sensory neurons and spinal dorsal horn. The release of glutamate in the synaptic cleft activates the postsynaptic ionotropic receptors leading to the opening of ion channels and membrane depolarization. The NMDA receptor antagonism is responsible for the particular ketamine properties (amnesic and psycho-sensory effects, analgesia, and neuroprotection). ${ }^{19}$ Activation of NMDA receptors allows the passage of $\mathrm{Ca}^{2+}$ and $\mathrm{Na}+$ into the cell and $\mathrm{K}+$ out of the cell. Coactivation of the glycine and glutamate sites of the receptor and removal of $\mathrm{Mg}^{2+}$ from the receptor's channel by membrane depolarization is required for the activation NMDA receptor. ${ }^{20}$ Each NMDA receptor is composed of four subunits. Research has identified up to seven subunit types of NMDA receptor. ${ }^{21}$ Subunit composition and subcellular location determine NMDA receptor function..$^{21}$ Their typical locations are in the synapse or the peri- or extrasynaptic space. Synaptic and extrasynaptic NMDA receptors demonstrate opposing effects. Synaptic NMDA receptor activation promotes the synaptic formation and neuronal survival while extrasynaptic NMDA receptor activation alters nuclear calcium to promote synaptic atrophy and neuronal death. ${ }^{22}$ Extrasynaptic NMDA receptor activation leads to uncontrolled target gene expression thus leading to reduced dendritic length and arborization, mitochondrial dysfunction, and loss of synapses. The distinct features of synaptic and extrasynaptic NMDA receptors facilitate the development of extrasynaptic NMDA receptor modulators. For example, synaptic NMDA receptors predominantly contain GluN2A and are coactivated primarily by D-serine whereas extrasynaptic NMDA receptors have abundant GluN2B subunits and are mainly coactivated by glycine. ${ }^{22}$ There are some glutamateindependent mechanism of ketamine that involves the interaction of ketamine with many binding sites such as cholinergic, opioid, monoaminergic, nicotinic, and muscarinic receptors. GammaAmino-Butyric Acid (GABA) is the most prevalent inhibiting neurotransmitter, responsible for an increase of chlorine conductance. Ketamine potentializes the GABA inhibition (GABA-A complex), but this interaction does not account for the analgesic effects. ${ }^{23}$ Ketamine agonism on spinal GABA receptors plays a role in spinal analgesia obtained with very high concentrations of ketamine (more than $500 \mathrm{IM}$ ) that is clinically not feasible. ${ }^{23}$ Ketamine binds to mu, delta, and kappa opioid receptors. The interaction of ketamine with opioid receptors is not responsible for the analgesic effect since the analgesic effect is not antagonized by naloxone. ${ }^{24}$ Ketamine creates a hyperadrenergic state by stimulating noradrenergic neurons and inhibition of catecholamines uptake. Because of its interaction with the serotonin transporter, Ketamine also inhibits dopamine and serotonin uptake. ${ }^{25}$ Ketamine's serotoninergic mechanism is also evidenced by the inhibition of ketamine emetic properties with ondansetron. ${ }^{26}$ These interactions involving noradrenergic neurons are implicated in the hypnotic, psychic, and analgesic effects of the ketamine molecule. Psychodysleptic effects could be initiated by L-type calcium channels which can be antagonized by nimodipine. ${ }^{27}$

\section{Synaptic and neuronal basis of ketamine action}

The is no single known basis of ketamine action for synaptic and neuronal effects. There are two possible mechanisms by which ketamine elicit its neuronal action. The first mechanism is that NMDA receptors present on inhibitory interneurons are tonically active and thus drive inhibition onto excitatory networks. NMDA receptor blockade leads to a decrease in the activity of these interneurons and ultimately facilitates excitatory networks. ${ }^{28}$ The second possible mechanism ketamine triggers an antidepressant response suggests a more synapse-specific effect of ketamine as the basis for its rapid behavioral effect. Studies suggest that NMDA receptor block by lowdose ketamine results in particular effects on downstream intracellular signaling. Blockade of spontaneous NMDA receptors results in inhibition of eukaryotic elongation factor (eEF2) kinase ${ }^{29}$ and a resulting decrease in phosphorylation of eEF2 that facilitates protein translation leading to an upregulation of brain-derived neurotrophic 
factor (BDNF) that triggers insertion of AMPA receptors and other traditional synaptic plasticity processes. These studies demonstrated that pharmacologically inhibiting the eEF2 kinase was sufficient to trigger a rapid and long-lasting antidepressant response independent of blocking NMDA receptors. ${ }^{28}$

\section{Neurobiology of depression relevant to ketamine action}

The term synaptic plasticity indicates the mechanisms through which neural circuits regulate their excitability and connectivity, particularly in the context of adaptation, e.g., processes of development, learning, coping with stress, and aging. ${ }^{29}$ These phenomena are accomplished by regulating synaptic strength [e.g., changing the number of $\alpha$-amino-3-hydroxy-5-methyl-4-isoxazolepropionic acid (AMPA) receptors] and synaptic number (e.g., altering dendritic spine density and shape). Synaptic plasticity can be long-term potentiation, or homeostatic plasticity. ${ }^{30}$ Homeostatic plasticity is associated with reduced prefrontal synaptic connectivity that is related to clinical depression. Synaptic scaling- a major form of homeostatic plasticity controls the overall strength of neuronal synaptic connectivity. Synaptic scaling is regulated by the prolonged increase in neuronal activities, inflammatory cytokines (eg. TNF) and neurotrophins (eg. BDNF).

\section{Synaptogenesis and the rapid antidepressant effects of ketamine}

Ketamine's antagonism of the glutamatergic NMDA receptor is the first step in a cascade of events that includes rapid increases in presynaptic glutamate release, enhanced regional activity in excitatory networks, and ultimately marked changes in synaptic plasticity and connectivity. A series of studies showed that low-dose ketamine administration triggers three consecutive events: a presynaptic disinhibition of glutamatergic neurons, increased activation of the AMPA glutamate receptor, combined with the blockade of extrasynaptic NMDA -receptors, and postsynaptic activation of neuroplasticity-related signaling pathways involving BDNF and mammalian target of rapamycin complex 1 (mTORC1), which results in overall synaptogenesis and synaptic potentiation. ${ }^{31}$

\section{Clinical biomarkers and the rapid antidepressant effects of ketamine}

Several biological measures have been utilized in clinical studies to characterize treatment response and to gain insight into neural substrates underlying ketamine's rapid antidepressant effects. These biomarkers can be clustered into three categories:

1. Biomarkers of synaptic strength and prefrontal excitability.

2. Biomarkers of restoration of neurotrophic function

3. Biomarkers of ketamine-induced glutamate surge.

\section{Synaptic strength and prefrontal excitability}

Magnetoencephalography studies found higher antidepressant responses to ketamine in depressed patients with pretreatment high rostral anterior cingulate activity in response to exposure to fearful faces. Conversely, high pregenual anterior cingulate activity during a working memory task predicted poor response to ketamine. ${ }^{32}$ Interestingly, patients with poor pretreatment connectivity between the pregenual anterior cingulate and the left amygdala showed a better response to ketamine treatment. Moreover, responders, but not nonresponders, to ketamine treatment showed increased stimulus-evoked somatosensory cortical excitability $\sim 6$ hours post treatment. This finding was interpreted as evidence of a positive relationship between ketamine-induced synaptic potentiation and response to treatment. ${ }^{32}$ Additional evidence associating synaptic potentiation with ketamine antidepressant effects comes from an electroencephalography (EEG) study, which examined sleep slow waves as putative markers of synaptic plasticity. The study provided evidence of increased synaptic strength the night after ketamine treatment of depressed patients. ${ }^{32}$

\section{Restoration of neurotrophic function}

Following treatment with ketamine, serum levels of BDNF was significantly reduced in nonresponders. ${ }^{33}$ A more recent, relatively large trial did find a positive relationship between peripheral BDNF levels and the antidepressant effect of ketamine in depressed patients. Four hours post-ketamine infusion, plasma BDNF increased in responders compared to nonresponders. ${ }^{34}$ Using EEG sleep slow waves as a marker of plasticity, another clinical study found a positive relationship between enhanced synaptic strength and increased plasma BDNF 4 hours post-ketamine administration.

\section{Ketamine-induced glutamate surge}

The presence of a glutamate surge and its association with the psychotomimetic effects of ketamine in humans has been demonstrated by many studies. ${ }^{35}$ They showed that glutamate release inhibitors, such as lamotrigine or group II metabotropic agonists, attenuate the perceptual effects. ${ }^{35}$ Altered neurotransmission of showed increased neural metabolism, particularly in the PFC, following the administration of low-dose ketamine ${ }^{35}$

\section{Cardiovascular and hepatic effects of ketamine}

Ketamine has an indirect stimulatory effect and a direct negative inotropic effect on the cardiovascular system. ${ }^{36}$ Stimulation is due to activation of the sympathetic system and is associated with the systemic release of catecholamines, norepinephrine release from sympathetic ganglia, inhibition of the vagal nerve, and the inhibition of norepinephrine reuptake at non-neuronal tissues such as the myocardium and peripheral nerves. Myocardial depression is evident after repeated (within minutes to hours) dosing of ketamine or high-dose ketamine infusion. Low dose ketamine infusion leads to cardiovascular stimulation characterized by tachycardia, systemic and pulmonary hypertension, and increases in cardiac output and myocardial oxygen consumption. ${ }^{36}$ So monitoring is required when treating chronic pain patients with cardiovascular disease with lowdose ketamine. Treatment with $\beta$-adrenoceptor blockade or clonidine improves hemodynamics following ketamine treatment.

\section{Hepatic ketamine effects}

Some reports are showing an elevated liver enzyme profile following anesthetic and subanaesthetic ketamine treatment. ${ }^{36}$ For example, a study by Noppers etal. ${ }^{37}$ observed that the second exposure to S-ketamine just three weeks following a $100 \mathrm{~h}$ treatment in CRPS-1 patients caused a very high liver enzyme elevation to a dangerous level. In three of six treated patient's alkaline phosphatase, aspartate transaminase, alanine transaminase, and $\gamma$-glutamyl transferase increased three times over the upper limit of normal. Upon termination of the ketamine infusion, the enzymes slowly returned to 
normal (normal values reached within three months).

\section{Ketamine cystitis:A serious adverse effect of ketamine}

Extreme ketamine use can cause Ketamine cystitis (KC) which is an injury the bladder, causing wounds (ulcers) and fibrosis (shrinkage and stiffening of the bladder walls). Patients often struggle with urinary frequency, urgency, pressure, pain, incontinence and bleeding from the bladder. ${ }^{38}$ They are often diagnosed to have ulcerative bladder, interstitial cystitis or ketamine cystitis.

\section{Epidemiology of ketamine cystitis}

The precise prevalence of recreational use of ketamine is unknown to obtain the exact data on ulcerative cystitis. A study done in Taiwan has indicated a dramatic increase in ketamine use in the past few years. It also reported the abuse of ketamine by young population with an age less than a median of 30 years. ${ }^{38}$ In Australia, lifetime use of ketamine was reported by $1 \%$ of Australians aged 14 years or older, with $0.3 \%$ reporting recent use. ${ }^{38}$ Prevalence of ketamine use was highest among those aged 20-29 years. ${ }^{38}$ Only a few people used ketamine in their 40s.38 A recent study in a large cohort of nontreatment-seeking ketamine users to assess the prevalence of urinary symptoms in revealed that $26.6 \%$ of recent ketamine users experienced urinary symptoms 39 . Urinary symptoms were greatly related to both doses of ketamine used and frequency of ketamine use..$^{38}$ Currently, no gender bias has been found in ketamine-associated cystitis (KC), although some series have reported a slight male predominance in ketamine abuse.

\section{Pathophysiology of ketamine cystitis}

The pathogenesis of bladder dysfunction could result from the two active metabolites of ketamine: norketamine and hydroxynorketamine. Recent studies have shown that ketamine and its active metabolites can be measured in high quantities in the urine of patients using ketamine. It is conceivable that ketamine and its active metabolites may accumulate in the urine and induce significant bladder irritation. Studies postulated four pathophysiological mechanisms that might account for the urinary tract damage. The first postulation is that presence of high concentration of ketamine and its metabolites in the urine might cause direct toxic effects on the urinary bladder interstitial cells, causing a chronic submucosal inflammatory response. The second one postulates that ketamine and its metabolites might induce microvascular changes in the urinary bladder and possibly the kidney, causing endothelial cell injury of microvessels, leading to compromised intrinsic microcirculation, or decreased microvascular density in the subendothelium. The third postulation is that an autoimmune reaction to the bladder urothelium and submucosa triggered by the presence of circulating ketamine or urinary ketamine and its metabolites. Finally, the fourth postulation is that these patients are unlikely to have bacteriuria as the possible cause of cystitis and papillary necrosis compared with other possible causes as described above. ${ }^{39}$ Recent pathological research on the urinary bladder of ketamine addiction employing mice reveals mononuclear infiltration, similar to that of the clinical situation of interstitial cystitis/painful bladder syndrome. There was also a possible decrease in the cholinergic neurons in the urinary bladder of the ketamine treated animals. A mouse model of ketamine abuse was developed recently and showed that dysregulation of purinergic neurotransmission might be the cause of detrusor overactivity in ketamine-induced bladder dysfunction. Chuang ${ }^{40}$ and colleagues state that ketamine also initiated the upregulation of COX-
2, iNOS and eNOS expression, which may play an important role in contributing to ketamine-induced alterations in micturition patterns and ulcerative cystitis. Bladder tissue from $\mathrm{KC}$ and Interstitial Cystitis (IC) patients found to have similar characteristics of the defective junctional protein, increased urothelial cell apoptosis, and increased suburothelial inflammation. ${ }^{41}$ Decreased expression of E-cadherin, a $\mathrm{Ca} 2+$-dependent, transmembrane cell adhesion molecule, and the increase of apoptosis was more severe in KC bladders than IC. ${ }^{41}$

\section{Clinical presentation}

A recent study has demonstrated that using ketamine for at least a two-year habit of three or more times a week is associated with lower urinary tract dysfunction and that these symptoms may persist for up to one year after the cessation of drug use. ${ }^{42}$ The symptoms of KC include a variety of Lower Urinary Tract Symptoms mainly irritative in nature. Typically, patients complain of nocturia, urgency, extreme frequency, bladder pain, and intractable dysuria. Gross hematuria is also a frequent symptom in those suffering from Ketamine cystitis. Another study ${ }^{43}$ reported that symptoms appeared after one month of starting the usage and became severe by the end of 1 year. Flank pain may occur due to hydronephrosis secondary to ureteral stricture or vesicoureteric reflux (VUR). ${ }^{42}$

\section{Investigation}

Symptom questionnaires may help to evaluate the severity of the symptomatology and monitor disease progression or regression with or without treatment, although they have not been validated as diagnostic criteria. Normally, urinalysis and urine cultures show nonbacterial pyuria on an initial assessment of the mid-stream urine sample. Cystoscopy reveals various degrees of epithelial inflammation and neovascularization of the bladder. Severe cases may present petechial hemorrhages and ulcers of the bladder mucosa, as classically described in patients with IC. Urodynamically, either detrusor overactivity or decreased bladder compliance with, or VUR was detected to some degree in those patients with $\mathrm{KC}$. It has been postulated that VUR is a secondary event to the severely contracted bladder with high detrusor pressure.

\section{Treatment}

The goal of treatment is to prevent deterioration of the renal function and offer the possibility of symptom resolution. Currently, ketamine cessation is the only effective treatment modality; however, the effect is likely to be dependent on the severity and duration of the use or abuse..$^{43}$ The bladder function of patients with near-normal cystometric bladder capacities has been shown to return to normal after stopping or reducing ketamine abuse. ${ }^{44}$ Patient compliance has previously been shown to be poor in patients suffering addiction and failure to abstain may lead to disease progression. Various treatment regimens have been used to treat the patients such as antibiotics, oral nonsteroidal anti-inflammatory drugs, steroids, anticholinergic therapy, and hydrodistention of the bladder; ${ }^{45}$ however, all have failed to provide significant and lasting improvement. It is still not clear whether the full symptomatic resolution will not be achieved in more advanced cases with severely reduced bladder capacity and compliance and consequent hydronephrosis. Because the clinical features of ketamine-associated cystitis are similar to IC, the possible etiology of impaired epithelial impermeability leading to leakage of the glycosaminoglycan layer has led to the use of oral pentosan polysulfate (Elmiron) and intravesical instillation of hyaluronic acid (Cystistat) in 
the treatment of IC. A low molecular weight heparin-like compoundElmiron, has been found useful to rebuild the glycosaminoglycan layer of the damaged urothelium, and a few patients experienced symptom relief after treatment. ${ }^{38}$ Tsai et al. ${ }^{38}$ have reported that all patients who had hyaluronic acid instillation had symptomatic relief, especially in relieving the bladder pain, frequency, and hematuria, but long-term follow-up is needed. It is unclear whether it is purely attributable to the abstinence, therapy, or both. These two options may provide a sense of direction, but longer follow-up is needed. If conservative treatment fails to relieve the symptoms and preserve renal function, augmentation enterocystoplasty or cystectomy with conduit diversion should be considered. ${ }^{38}$ However, if the patient continues to abuse ketamine, they should be warned that fibrotic change of the urinary reservoir may occur, and that may lead to severe and irreversible urological pathologies.

\section{Bladder instillation agents}

Dimethylsulfoxide (DMSO) is a liquid medication approved by the US Food and Drug Administration to treat interstitial cystitis/ bladder pain syndrome (IC/BPS). ${ }^{46} \mathrm{DMSO}$ is instilled into the bladder through a temporary catheter and is held in place for approximately 20 minutes. DMSO treatments are often given weekly, for six to eight weeks or longer. It has no known long-term side effects and has been used for many years and is considered to be very safe. Other bladder instillations: Some healthcare providers recommend a combination of medications instilled into the bladder with a catheter, to reduce symptoms of pain. This can be done in a clinician's office, or at home if the patient can learn to self-administer the treatment. The treatment may be used as a single "rescue" treatment when symptoms are severe or as a regularly scheduled treatment (e.g., three times per week for four weeks). The medications are in a liquid form and are a small amount (about $15 \mathrm{~mL}$ or 0.5 ounces). The patient holds the liquid in the bladder for as long as possible and then urinate normally. The combination of medications may include lidocaine, heparin, and sodium bicarbonate. ${ }^{47}$ It is believed that this combination helps to repair the bladder lining and decrease nerve sensitivity in the bladder. In one small study, approximately 80 percent of patients had decreased pain for at least four hours after one treatment. ${ }^{47}$ Also, some patients experience reduced pain for days or weeks after bladder installations.

\section{Conclusion}

Ketamine has been found to be very effective in managing depression in various stages, producing a rapid antidepressant effect. It is valuable in suicidal risk patients too. As of any psychotropic drug, ketamine has a high risk for abuse too.Chronic ketamine use has been associated with severe ketamine cystitis, which may lead to bladder contracture and dysfunction. The goal of treatment is to prevent deterioration of the renal function and indeed offer the possibility of symptom resolution. Currently, ketamine cessation is the only effective treatment modality, but the effect is likely to be dependent on the severity and duration of the use or abuse. A variety of drugs is found minimally useful in managing ketamine cystitis when used as instillation. Bladder instillation is inconvenient and leads to higher rate of non-compliance. DMSO is found to be useful in managing symptoms but requires patient training and supplies, definitely requires more resources than taking an oral medication. Further research should focus on drugs that can be taken orally to neutralize the metabolites of ketamine in the bladder. A multidisciplinary approach promoting the education of the youth to stop abuse, harm reduction, cessation, and early referral is needed to manage individuals with $\mathrm{KC}$ to avoid progression.

\section{Acknowledgements}

None.

\section{Conflict of interest}

The author declares that there is no conflict of interest.

\section{References}

1. Centers for Disease Control and Prevention. Depression in the U.S. Household Population, 2009-2012. 2014.

2. Grohol J. How Long Do Antidepressants Take to Work?. Psych Central. 2015.

3. Abdallah CG, Sanacora G, Duman RS, et al. Ketamine and Rapid-Acting Antidepressants: A Window into a New Neurobiology for Mood Disorder Therapeutics. Annual Review of Medicine. 2015;66:509-523.

4. Monteggia LM, Zarate C. Antidepressant actions of ketamine: from molecular mechanisms to clinical practice. Current Opinion in Neurobiology. 2015;30:139-143.

5. Nosyreva E, Szabla K, Autry AE, et al. Acute suppression of spontaneous neurotransmission drives synaptic potentiation. $J$ Neurosci. a2013;33(16):6990-7002.

6. Lee Y, Syeda K, Maruschak NA, et al. A New Perspective on the AntiSuicide Effects With Ketamine Treatment: A Procognitive Effect. $J$ Clin Psychopharmacol. 2016;36(1):50-56.

7. Zarate CA, Brutsche N, Laje G, et al. Relationship of Ketamine's Plasma Metabolites with Response, Diagnosis, and Side Effects in Major Depression. Biol Psychiatry. 2012;72(4):331-338.

8. Ibrahim L, Diazgranados N, Luckenbaugh DA, et al. Rapid Decrease in Depressive Symptoms with an N-methyl-D-aspartate Antagonist in ECTResistant Major Depression. Prog Neuropsychopharmacol Biol Psychiatry. 2012;35(4):1155-1159.

9. Hirota K, Lambert DG. Ketamine: New uses for an old drug? $\mathrm{Br} \mathrm{J}$ Anaesth. 2011;107(2):123-126.

10. Gorlin AW, Rosenfeld DM, Ramakrishna H. Intravenous sub-anesthetic ketamine for perioperative analgesia. Journal of Anaesthesiology, Clinical Pharmacology. 2016;32(2):160-167.

11. Niesters M, Martini C, Dahan A. Ketamine for chronic pain: risks and benefits. Br J Clin Pharmacol. 2014;77(2):357-367.

12. Meng E, Wu S, Cha T, et al. A murderer of young bladders: Ketamineassociated cystitis. Urological Science. 2013;24(4):113-116.

13. Mion G, Villevieille T. Ketamine pharmacology: an update (pharmacodynamics and molecular aspects, recent findings). CNS Neurosci Ther. 2013;19(6):370-380.

14. Li Y, Coller JK, Hutchinson MR, et al. The CYP2B6*6 Allele Significantly Alters the N-Demethylation of Ketamine Enantiomers In Vitro. Drug Metab Dispos. 2013;41(6):1264-1272.

15. Peltoniemi MA, Hagelberg NM, Olkkola KT, et al. Ketamine: A Review of Clinical Pharmacokinetics and Pharmacodynamics in Anesthesia and Pain Therapy. Clin Pharmacokinet. 2016;55(9):1059-1077.

16. Chen JT, Chen RM. Mechanisms of ketamine-involved regulation of cytochrome P450 gene expression. Expert Opin Drug Metab Toxicol. 2010;6(3):273-281. 
17. Kurdi MS, Theerth KA, Deva RS. Ketamine: Current applications in anesthesia, pain, and critical care. Anesth Essays Res. 2014;8(3):283-290.

18. Bojak I, Day HC, Liley DTJ. Ketamine, Propofol, and the EEG: A Neural Field Analysis of HCN1-Mediated Interactions. Front. Comput. Neurosci. 2013;7:22.

19. Duman RS, Li N, Liu RJ, et al. Signaling Pathways Underlying the Rapid Antidepressant Actions of Ketamine. Neuropharmacology. 2012;62(1):3541 .

20. Mayer ML. Structural biology of glutamate receptor ion channel complexes. Curr Opin Struct Biol. 2016;41:119-127.

21. Paoletti P, Bellone C, Zhou Q. NMDA receptor subunit diversity: Impact on receptor properties, synaptic plasticity and disease. Nat Rev Neurosci. 2013;14(6):383-400.

22. Zhou X, Chen Z, Yun W, et al. Revisiting the function of extrasynaptic NMDA receptor in excitotoxicity Running title: NMDA receptor and excitotoxicity. Neuroscientist. 2015;21(4):337-344.

23. Rosa PB, Neis VB, Ribeiro CM, et al. Antidepressant-like effects of ascorbic acid and ketamine involve modulation of GABAA and GABAB receptors. Pharmacol Rep. 2016;68(5):996-1001.

24. Garg A, Sinha P, Kumar P, et al. Use of naltrexone in ketamine dependence. Addictive Behaviors. 2014;39(8):1215-1216.

25. Khezri MB, Ghasemi J, Mohammadi N. Evaluation of the analgesic effect of ketamine as an additive to intrathecal bupivacaine in patients undergoing cesarean section. Acta Anaesthesiologica Taiwanica. 2013;51(4):155-160.

26. Lee JS, Jeon WC, Park EJ, et al. Does ondansetron have an effect on intramuscular ketamine-associated vomiting in children? A prospective, randomised, open, controlled study. $J$ Paediatr Child Health. 2014;50(7):557-561.

27. Jahromi SA, Masoumeh S, Hatamian S. Comparison Between Effect of Lidocaine, Morphine and Ketamine Spray on Post-Tonsillectomy Pain in Children. Anesth Pain Med. 2012;21(2):17-21.

28. Autry AE, Adachi M, Nosyreva E, et al. NMDA Receptor Blockade at Rest Triggers Rapid Behavioural Antidepressant Responses. Nature. 2011;475(7354): 91-95.

29. Maus M, Torrens Y, Gauchy C, et al. 2-Deoxyglucose and NMDA inhibit protein synthesis in neurons and regulate phosphorylation of elongation factor-2 by distinct mechanisms. J Neurochem. 2006;96(3):815-824.

30. Turrigiano G. Homeostatic Synaptic Plasticity: Local and Global Mechanisms for Stabilizing Neuronal Function. Cold Spring Harb Perspect Biol. 2012;4(1):a005736.

31. Liu RJ, Lee FS, Li XY, et al. BDNF Val66Met allele impairs basal and ketamine-stimulated synaptogenesis in prefrontal cortex. Biol Psychiatry. 2012;71(11):996-1005.

32. Cornwell BR, Salvadore G, Furey M, et al. Synaptic potentiation is critical for rapid antidepressant response to ketamine in treatment-resistant major depression. Biol Psychiatry. 2012;72(7):555-561.
33. Duncan WC, Sarasso S, Ferrarelli F, et al. Concomitant BDNF and sleep slow wave changes indicate ketamine-induced plasticity in major depressive disorder. Int J Neuropsychopharmacol. 2013;16(2):301-311.

34. Haile $\mathrm{CN}$, Murrough JW, Iosifescu DV, et al. Plasma brain derived neurotrophic factor (BDNF) and response to ketamine in treatmentresistant depression. Int J Neuropsychopharmacol. 2012;17(2):331-336.

35. Luckenbaugh DA, Niciu MJ, Ionescu DF, et al. Do the dissociative side effects of ketamine mediate its antidepressant effects? J Affect Disord. 2014;159:56-61.

36. Chowdhury GM, Behar KL, Cho W, et al. ${ }^{1} \mathrm{H}-\left[{ }^{13} \mathrm{C}\right]-\mathrm{NMR}$ Spectroscopy Measures of Ketamine's Effect on Amino Acid Neurotransmitter Metabolism. Biological Psychiatry. 2012;71(11):1022-1025.

37. Dwyer JM, Lepack AE, Duman RS. mTOR Activation Is Required for the Antidepressant Effects of mGluR ${ }_{2 / 3}$ Blockade. The International Journal of Neuropsychopharmacology/Official Scientific Journal of the Collegium Internationale Neuropsychopharmacologicum (CINP). 2012;15(4):429434.

38. Meng E, Wu S, Cha T, et al. A murderer of young bladders: Ketamineassociated cystitis. Urological Science. 2013;24(4):113-116.

39. Clemens JQ. The prevalence and natural history of urinary symptoms among recreational ketamine users. BJU Int. 2013;53-54.

40. Chuang S, Liu K, Li Y, et al. Dual involvements of cyclooxygenase and nitric oxide synthase expressions in ketamine-induced ulcerative cystitis in rat bladder. Neurourol Urodyn. 2013;32(8):1137-1143.

41. Lee C, Shie J, Wang Y, et al. Increased Suburothelial Inflammation, Increased Urothelial Cell Apoptosis And Decreased E-Cadherin Intensity In The Ketamine Associated Bladder Dysfunction- An Immunohistochemistry Comparison With Interstitial Cystitis. The Journal of Urology. 2012;187(4):e45-e46.

42. Huang P, Meng E, Cha T, et al. 'Walking-stick ureters' in ketamine abuse. Kidney International. 2011;80(8):895.

43. Yee C, Ma W, Ng C, et al. Ketamine-Associated Uropathy: From Presentation to Management. Curr Bladder Dysfunct Rep Current Bladder Dysfunction Reports. 2016;11(3):266-271.

44. García-Larrosa A, Castillo C, Ventura M, et al. Cystitis and ketamineassociated bladder dysfunction. Actas Urológicas Esp. 2012;36(1):60-64.

45. Wei Y, Yang J. Ketamine-induced ulcerative cystitis' is perhaps better labelled 'ketamine-induced uropathy'. Addiction. 2015;108(8):1515-1515.

46. Tomoe H. In what type of interstitial cystitis/bladder pain syndrome is DMSO intravesical instillation therapy effective? Transl Androl Urol. 2015;4(6):600-604.

47. Tsai YC, Kuo H. Ketamine cystitis: Its urological impact and management. Urological Science. 2015;26(3):153-157. 